\title{
Critical appraisal of canakinumab in the treatment of adults and children with cryopyrin-associated periodic syndrome (CAPS)
}

This article was published in the following Dove Press journal:

Biologics: Targets \& Therapy

14 May 2010

Number of times this article has been viewed

\author{
Ori Toker' \\ Philip J Hashkes ${ }^{2}$ \\ 'Department of Pediatrics, Shaare \\ Zedek Medical Center, affiliated \\ with the Hadassah-Hebrew University \\ Medical School, Jerusalem, Israel; \\ ${ }^{2}$ Pediatric Rheumatology Unit, Shaare \\ Zedek Medical Center, Jerusalem, \\ Israel, Cleveland Clinic Lerner Medical \\ School, Case Western Reserve \\ University, Cleveland, OH, USA
}

\begin{abstract}
The cryopyrin-associated syndromes (CAPS) include three autosomal-dominant syndromes, that are caused by a mutation in the NLRP3 gene on chromosome 1, encoding the cryopyrin protein. These syndromes, familial cold autoinflammatory syndrome, Muckle-Wells syndrome and neonatal-onset multisystem inflammatory disease, are characterized by urticaria-like rash, fever, central nervous system inflammation, an arthropathy and a risk of the development of amyloidosis in a respectively escalating degree of severity between the various syndromes. Recently the role of cryopyrin in the regulation of interleukin (IL)-1 production and activation was described and anti IL-1 therapies were found to be very effective in treating these syndromes. There are several types of anti IL-1 medications based on different mechanisms of antagonizing IL-1. This paper focuses on the efficacy and safety of canakinumab, a long-acting humanized anti IL-1 antibody, in treating these syndromes.
\end{abstract}

Keywords: canakinumab, cryopyrin-associated periodic syndromes, biologics, treatment, autoinflammatory diseases

\section{Introduction to cryopyrin-associated periodic syndrome and prevalence}

The cryopyrin-associated periodic syndromes (CAPS) include three autosomaldominant syndromes, that are caused by a mutation in the NLRP3 (in past also called CIAS1/NALP3) gene on chromosome 1, encoding the cryopyrin protein (Table 1a). The three syndromes, familial cold autoinflammatory syndrome (FCAS), Muckle-Wells syndrome (MWS) and neonatal-onset multisystem inflammatory disease (NOMID), are characterized by urticaria-like rash, fever, central nervous system inflammation, arthropathy and the risk of later development of amyloidosis(Table 1b, 1c). These syndromes are considered variants of the same disease process that vary by severity of symptoms, systems involved and outcome. ${ }^{1}$ All respond dramatically to anti-interleukin (IL)-1 therapy.

The prevalence of CAPS in the United States (US) is estimated at about $1 / 10^{6}$, although there are no formal studies and it is probable there is underdiagnosis of CAPS, especially the MWS variant.

\section{Familial cold autoinflammatory syndrome}

FCAS (previously called familial cold urticaria), the mildest of the CAPS, was first described in 1940 and the genetic mutation discovered in 2001..$^{2,3}$ Most FCAS patients are located in the US and there are probably several hundred patients with this disorder. It has been hypothesized that most patients originate from the same family. Almost all 
patients have a genetic mutation in the NLRP3 gene. FCAS often starts at birth and is apparent in $95 \%$ of the patients by 6 months.

\section{Clinical manifestations ${ }^{4}$}

Typically, the attack starts 2 to 3 hours after generalized (not direct contact) cold exposure. Patients develop an urticaria-like rash that starts on the extremities and spreads to the trunk, low-grade fever, arthralgia, conjunctivitis, nausea, extreme thirst, sweating and headaches. The peak of the attack occurs at 6 to 8 hours and lasts up to 24 hours. The frequency of attacks is variable but can be very frequent and debilitating. The rash is not true urticaria (mast cells), rather there is a perivascular polymorphonuclear cellular infiltrate in the dermis. Amyloidosis is a rare complication of FCAS ( $2 \%$ to $4 \%)$.

\section{Muckle-Wells syndrome}

MWS, the intermediate severity CAPS, was described in 1962 and the genetic mutation was found together with that of FCAS. ${ }^{3,5}$ MWS starts later in life than FCAS and can appear at any age. Most cases are in Europe. Genetic mutations in the $N L R P 3$ gene are found in $65 \%$ to $75 \%$ of patients.

\section{Clinical manifestations ${ }^{6}$}

Attacks are usually not triggered by cold exposure. A typical attack includes fever, rash, more persistent than in FCAS, arthralgia, arthritis, myalgia, headaches, conjunctivitis, episcleritis and uveitis. Attacks last up to 3 days. Often starting in adolescence, $50 \%$ to $70 \%$ of patients develop sensorineural hearing loss. Amyloidosis develops in $25 \%$ of the patients.

\section{Neonatal-onset multisystem inflammatory disease}

NOMID, termed in Europe chronic infantile neurologic, cutaneous, articular syndrome (CINCA), is the most severe of the CAPS. First described in 1981, the association with the NLRP3 gene was found in 2002. ${ }^{7,8}$ However, only about $50 \%$ to $60 \%$ of patients have mutations in this gene. ${ }^{9}$

\section{Clinical manifestations $\mathbf{s}^{1,7,9}$}

The onset of NOMID is at or within several weeks of birth; $33 \%$ to $50 \%$ of affected infants are born prematurely. Patients present with urticaria-like rash and fever, often occurring daily. They also have symptoms related to chronic aseptic meningitis such as headaches, irritability and vomiting.

Table Ia Genetic characteristics of the cryopyrin-associated periodic syndromes

\begin{tabular}{llllll}
\hline Disease & Inheritance & Chromosome & Gene defect & Protein product & Common mutations \\
\hline FCAS & Dominant & $\mathrm{Iq} 44$ & CIASI/NALP3/NLRP3 & Cryopyrin & VI98M, C259W, R260W \\
MWS & Dominant & $\mathrm{Iq} 44$ & CIASI/NALP3/NLRP3 & Cryopyrin & R260W, L, L264V, D303N \\
NOMID & Dominant & $\mathrm{Iq} 44$ & CIASI/NALP3/NLRP3 & Cryopyrin & CI48Q, RI68Q, L264F, H, \\
& Sporadic & & & & RD303H, V35IM, L \\
\hline
\end{tabular}

Genetic mutations are found in only $50 \%$ to $70 \%$ of patients with MWS and NOMID.

Table Ib Characteristic of the cryopyrin-associated periodic syndromes

\begin{tabular}{llllll}
\hline Disease & Onset age & Typical ethnicity & Common triggers & Episode duration & $\begin{array}{l}\text { Interval between } \\
\text { episodes }\end{array}$ \\
\hline FCAS & First year of life & United States & Cold & $<24$ hours & Variable \\
MWS & Any age & Western Europe & Any & I-3 days & Variable \\
NOMID & Birth, infancy & Any & None & Continuous & None \\
\hline
\end{tabular}

Table Ic System involvement of the cryopyrin-associated periodic syndromes

\begin{tabular}{lllll}
\hline Disease & Skin & Musculoskeletal & Eyes & Neurologic \\
\hline FCAS & Urticaria-like & Arthralgia & $\begin{array}{l}\text { Conjunctivitis } \\
\text { Conjunctivitis, } \\
\text { episcleritis, uveitis }\end{array}$ & Headaches \\
MWS & Urticaria-like & Arthralgia & $\begin{array}{l}\text { Conjunctivitis, } \\
\text { uveitis, papillitis }\end{array}$ & $\begin{array}{l}\text { Chronic meningitis, hearing loss, } \\
\text { mental retardation increased } \\
\text { intracranial pressure, headaches }\end{array}$ \\
\hline
\end{tabular}

Abbreviations: FCAS, familial cold autoinflammatory syndrome; MWS, Muckle-Wells syndrome; NOMID, neonatal-onset multisystem inflammatory disorder. 
Late neurologic complications include hydrocephalus, developmental delay, mental retardation and hearing loss. Eye findings include conjunctivitis, uveitis and papillitis of the optic nerve, and may lead to visual loss. By about 2 years, $50 \%$ of patients develop a severe arthropathy, consistently mainly of cartilage growth abnormalities leading to severe pain, metaphyseal and epiphyseal bony overgrowth, ossification irregularities, deformities and disabilities. There is little synovial inflammation in NOMID. Patients have typical morphological changes of short stature, frontal bossing, macrocephaly, saddle nose, short, thick extremities with clubbing of fingers and wrinkled skin. About $20 \%$ die by age 20 years and others develop amyloidosis.

\section{Etiology and pathogenesis of CAPS}

The three autosomal-dominant syndromes that constitute CAPS are caused by single base mutations on the NLRP3 gene located on the long arm of chromosome 1 encoding the protein cryopyrin. ${ }^{1,3,8}$ Some mutations are specific to one of the syndromes and some overlap among all three syndromes (Table 1a). ${ }^{1,9}$ Often, especially in patients with NOMID, no mutations are found, thus it is clear that other factors influence the development and severity of CAPS. ${ }^{9}$

The cryopyrin protein contains an N-terminal pyrin domain and has an important role in regulation of the assembly of the inflammasome, a group of proteins that when assembled activate caspase- 1 that cleaves pro IL- $1 \beta$ to active IL- $1 \beta .{ }^{10}$ The mutated cryopyrin protein probably increases the rate of the inflammasome assembly independently of the usual stimuli needed in wild type protein. ${ }^{10,11}$ Thus the result is a gain of function mutation. Cryopyrin is present mainly in neutrophil cells and chondrocytes explaining the target organs of these diseases.

\section{Management}

Non-steroidal anti-inflammatory drugs (NSAIDs) and anti-histamine therapies are not effective for FCAS and MWS. Corticosteroids may alleviate symptoms but are needed frequently and may cause significant adverse effects. Anti-IL-1 agents are very effective in alleviating symptoms and decreasing inflammation. ${ }^{6,12-18}$ In recent controlled trials, rilonacept a fusion protein soluble IL-1 receptor, and canakinumab, a humanized IL-1 antibody, have been shown to be effective in treating FCAS and MWS. ${ }^{19,20}$ In about one-third of cases of MWS the hearing loss may be partially reversible. ${ }^{21-23}$

In NOMID, NSAIDs, antihistamines, corticosteroids, colchicine, methotrexate and other immunosuppressive medications are marginally effective and do not change the disease course. One case report showed that etanercept, a tumor necrosis factor soluble receptor, was effective, mainly for the joints. ${ }^{24}$

Recent studies have shown the dramatic effect of anakinra, a recombinant IL-1 receptor antagonist in treating the rash, fever and meningitis of NOMID with normalization of the acute phase reactants allowing a reduction in the corticosteroid dose. ${ }^{25-28}$ However, existing joint damage and mental retardation was not reversible and many patients continue to have increased intracranial pressure despite therapy. It appears that early recognition and treatment is crucial in preventing long-term damage and disability.

\section{Canakinumab in CAPS - mode of action}

Canakinumab (ACZ885, Ilaris ${ }^{\circledR}$; Novartis Pharma) is a recombinant, long-acting humanized anti-IL-1 $\beta$ monoclonal antibody, which selectively blocks IL-1 $\beta$. Canakinumab also appears to have intracellular effects in addition to its' primary effect as a neutralizing antibody to IL-1. Lachmann et al demonstrated that the production of IL-1 $\beta$, which is markedly increased in CAPS patients (31 ng/day mean production examined in 7 patients), was reduced to production levels of healthy controls after the administration of canakinumab (6 ng/day). ${ }^{29}$ Thus canakinumab also acts as a negative feedback on the production of IL-1 $\beta$ by the inflammasome. ${ }^{30}$

\section{Pharmacology/pharmacokinetics}

Canakinumab binds to serum IL-1 $\beta$ and neutralizes its activity by blocking its interaction with IL-1 receptors with peak serum concentration occurring approximately 7 days after subcutaneous administration of a single, $150 \mathrm{mg}$ dose. The range of observed half-life was 21.5 to 33 days ${ }^{31,32}$ with a mean of 26 days. $^{30,31}$ The absolute bioavailability was $70 \%$ and the expected accumulation ratio was 1.3-fold following 6 months of subcutaneous dosing of $150 \mathrm{mg}$ administered every 8 weeks. Clearance of canakinumab varied according to body weight and was estimated to be $0.174 \mathrm{~L} /$ day in a CAPS patient weighing $70 \mathrm{~kg}$. There was no indication of accelerated clearance or time-dependent change in the pharmacokinetic properties of canakinumab following repeated administration. In pediatric CAPS patients, the peak concentrations of canakinumab occurred between 2 to 7 days following a single subcutaneous administration of $2 \mathrm{mg} / \mathrm{kg}$ ( $\max 150 \mathrm{mg}$ ) of canakinumab. The half-life ranged from 22.9 to 25.7 days, similar to the pharmacokinetic properties 
observed in adults. No gender- or age-related pharmacokinetic differences were observed after correction for body weight. $^{31}$

\section{Efficacy/comparative studies}

To date there are no published comparative studies, comparing canakinumab, anakinra or rilonacept in the treatment of CAPS. Lachmann et al published a pivotal, phase III doubleblind, placebo-controlled, randomized withdrawal study of canakinumab in patients with CAPS. ${ }^{20}$ Patients who had previously received treatment with anakinra or rilonacept, were eligible to participate after a minimal "washout" period when treatment was discontinued (different for each anti IL-1 medication based on half-life) and their disease had relapsed. $49 \%$ of the patients were treated with anakinra before entering the study, but there was no data on the efficacy or adverse effects of anakinra in these patients. Patients treated with canakinumab in previous preliminary trials were also allowed to participate when their disease flared. ${ }^{32,33}$ The study was designed as a three-part study. Overall 35 patients were enrolled, aged 4 to 75 years, weighing between $15-100 \mathrm{~kg}$. It is important to note that the authors did not present details on the proportion of patients with each subtype of CAPS. In the first part, patients received $150 \mathrm{mg}$ or $2 \mathrm{mg} / \mathrm{kg}$ (for patients $\leq 40 \mathrm{~kg}$ ) of open label canakinumab by a single, subcutaneous injection. Those with a complete response to treatment by day 15 who did not flare by the end of 8 weeks entered part 2 and were randomly assigned to receive either $150 \mathrm{mg}$ of canakinumab or placebo every 8 weeks for up to 24 weeks. After the completion of part 2 or at the time of relapse, whichever occurred first, patients proceeded to part 3 and received at least 2 more open-label doses of canakinumab.

The primary study outcome measure was the proportion of patients in part 2 with a relapse (Table 2) of CAPS during canakinumab treatment, as compared with placebo. Secondary outcome measures included the proportion of patients with a complete response in part 1 , the value of inflammatory markers (C-reactive protein, serum amyloid A), global assessments by physicians and patients and safety data. In the open-label first part, the symptoms of CAPS started to diminish in all patients within 24 hours and $97 \%$ of the patients had a complete response by day 15 . One patient did not maintain complete response and two other patients refused randomization and were excluded from part 2. Thirty-one patients were randomized. During the second part of the study, all 15 patients in the canakinumab group remained in remission. In contrast, 13 of the 16 patients (81\%) in the placebo group had a disease flare $(P<0.001)$, within a median of 100 days. The inflammatory markers remained within the normal range in the canakinumab group, but increased in the placebo group. At the final assessment at the end of part 3, a total of 30 of the $31(97 \%)$ patients who had entered this part of the study had no or minimal disease activity, according to the physician's assessment, and the remaining patient had mild disease activity. Rash was absent in 29 of the 31 patients (94\%) and was minimal in the other 2 patients. Either no or minimal symptoms were reported by 26 of the 31 patients $(84 \%)$, mild symptoms were reported by 1 patient, moderate symptoms by 2 patients, and severe symptoms by 1 patient, who also had fibromyalgia; data were missing for 1 patient.

A later multi-center open-label cohort study enrolled a total of 98 patients, including 18 pediatric patients. ${ }^{34}$ Fifty-four patients were included from various studies of Lachmann et al ${ }^{20,32,33}$ and 44 were canakinumab-naïve patients. The patients received $150 \mathrm{mg}$ or $2 \mathrm{mg} / \mathrm{kg}$ (for patients $\leq 40 \mathrm{~kg}$ ) of canakinumab every 8 weeks by subcutaneous injection. The primary outcome measure was to assess the long-term safety but secondary outcomes included assessment of response for canakinumab naïve patients, the maintenance of response for patients already receiving canakinumab and the percentage of patients requiring dose adjustment. The complete response for canakinumab-naïve patients was defined as in Table 2. A complete response was observed in 41 of the 44 canakinumab-naïve patients (93.2\%). Relapse data were available

Table 2 Descriptions of response in pivotal and other canakinumab studies in cryopyrin-associated periodic syndromes (CAPS)

\begin{tabular}{llll}
\hline & Disease activity assessments & Rash & Laboratory activity markers $^{\mathbf{b}}$ \\
\hline Complete response & no or minimal disease activity & no or minimal \\
Relapse & greater than minimal & more than minimal & within normal range \\
\hline
\end{tabular}

aisease activity assessments were performed monthly by physicians who assessed the global disease activity and each of the following symptoms: urticarial rash, arthralgia, myalgia, headache, conjunctivitis, fatigue or malaise, and other symptoms related or unrelated to CAPS, The assessments were performed with the use of a 5-point scale. ${ }^{b}$ The values for serum C-reactive protein and serum amyloid $A$ that was within the normal range ( $<10 \mathrm{mg} / \mathrm{L}$ for both measures) or defined as relapse when the value for either was greater than $30 \mathrm{mg} / \mathrm{L}$. 
for $86 \%$ of the patients; $90.6 \%$ of these had no relapse, and $5.9 \%$ had experienced at least 1 relapse. At least 1 dose adjustment was required by $16.3 \%$ of the patients (the dose adjustment was not described in the abstract).

In the pediatric subgroup of this study $(n=18$; range 5 to 17 years; 8 adolescents [ $\geq 12$ years]; all were $\geq 15 \mathrm{~kg}$ ), 11 were naïve to canakinumab and 7 were rolled over from Lachmann's et al study. ${ }^{35}$ Complete response was achieved in $9(81.8 \%)$ canakinumab-naïve patients by day 8 . The majority of canakinumab-treated pediatric patients were relapse free (11 out of 18). Three MWS patients experienced 1 relapse, 3 had missing relapse assessments and 1 MWS/NOMID overlap patient did not achieve complete response and the dose was uptitrated. Seven children $(36.8 \%)$ received at least 1 protocol-defined dose adjustment (first dose doubled) or at least 1 frequency adjustment. In other studies of canakinumab the median effect of one $150 \mathrm{mg}$ dose of canakinumab was 127 days. $^{29}$

As a result of the pivotal study results canakinumab was approved by the United States Food and Drug Administration in June 2009 for the treatment of FACS and MWS in adults and children 4 years of age and older (www.accessdata.fda. gov/drugsatfda_docs/label/2009/125319s000lbl.pdf), and in October 2009 by the European Medicines Agency for use in all subtypes of CAPS (www.ema.europa.eu/humandocs/ Humans/EPAR/ilaris/ilaris.htm).

However there are still important questions of efficacy not answered by these studies, particularly for MWS and NOMID associated with long-term damage. These include the prevention of amyloidosis, the prevention of arthropathy in NOMID, the prevention of neurologic damage, especially hearing loss in MWS and NOMID as well as ocular and other central nervous system (CNS) damage related to NOMID. Another unanswered question is whether existing damage can be reversed including amyloidosis, ${ }^{36}$ hearing loss, CNS function (mental retardation, for example) and the arthropathy associated with NOMID. Initial studies in anakinra indicate only limited ability to reverse existing damage. ${ }^{37}$

An important issue in answering these questions is whether there is adequate penetration of canakinumab to the CNS via the blood - brain barrier and whether current CNS levels are sufficient to treat these manifestations. As we noted, in Lachmann's studies it was not clear how many patients had classic NOMID and whether current doses and frequency of administration of canakinumab would be sufficient for these patients.

\section{Canakinumab effect on issues related to general health, fatigue, function and quality of life of CAPS patients}

Lachmann et al examined issues of general health, fatigue and function among the patients in the controlled phase III, 48 week study. ${ }^{38}$ Baseline scores for general mental and physical health measured by use of the Short-Form (SF)-36 ${ }^{\circledR}$ and fatigue measured by use of the Functional Assessment of Chronic Illness Therapy (FACIT)-Fatigue ${ }^{\circledR}$ questionnaires were significantly lower than those expected in the general population. Following the first canakinumab dose, SF-36 and FACIT-Fatigue $^{\circledR}$ scores improved to the levels expected in the general population (time to improvement not described). An improvement in functional disability was observed as shown by Health Assessment Questionnaire (HAQ) disability scores. Health-Related Quality of Life (HRQoL) scores at 48 weeks were comparable to those observed following the first injection, and to the general population, suggesting the sustained effect of therapy of canakinumab on primarily patient driven outcomes.

Thus, canakinumab for treatment of CAPS has shown a rapid and sustained significant impact on important physician- and patient-derived outcomes.

\section{Safety and tolerability}

Lachmann et al reported no deaths or life-threatening adverse effects (AE) in the pivotal study. ${ }^{20}$ Two patients had serious AEs in the open-label part 3, one a urinary tract infection requiring hospitalization and the other was an episode of vertigo accompanied by acute closed-angle glaucoma. Both patients discontinued therapy. Overall 29 (83\%) of the patients had AEs with 12 (34\%) developing infectious AEs. There was a significant difference between canakinumab and placebo only in the rate of suspected infections $(P=0.03)$. While numbers are small, vertigo was seen solely in canakinumab-treated patients with MWS who often have cochlear involvement as part of their disease. Table 3 describes the AEs seen most frequently in this cohort.

In various publications and abstracts safety data has been reported on 102 adult and pediatric patients with CAPS exposed to canakinumab..$^{20,29,32-35}$ These included 20 patients with FCAS, 72 MWS, 10 MWS/NOMID overlap. Sixty-two patients were treated for at least 6 months, 56 for at least 1 year and 4 for at least 3 years. A total of 9 serious AEs were reported. Among these were infections in 3 patients and vertigo in 2 . One of the infections included 
Table 3 Adverse events seen in more than 10\% of patients using canakinumab for cryopyrin-associated periodic syndromes: controlled study ${ }^{20}(\mathrm{~N}=35)$

\begin{tabular}{ll}
\hline Adverse event & N (\%) \\
\hline Nasopharyngitis & $12(34)$ \\
Diarrhea & $7(20)$ \\
Influenza & $6(17)$ \\
Rhinitis & $6(17)$ \\
Nausea & $4(14)$ \\
Headache & $5(14)$ \\
Pharyngitis & $5(14)$ \\
Weight gain & $4(11)$ \\
Musculoskeletal pain $_{\text {Vertigo }}^{a}$ & $4(11)$ \\
\hline
\end{tabular}

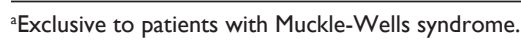

an intra-abdominal abscess following appendectomy. As in the controlled study, the most common AEs were nasopharyngitis and gastrointestinal disturbance. Other common AEs included influenza and headache (actual numbers of each AE unavailable from published data). There was no change in the AE profile among the longer treated patients. One patient discontinued treatment due to a potential infection. There were no cases of malignancy or opportunistic, including mycobacterium, infections reported. Also there were no cases of autoantibody formation to canakinumab or autoimmune/demyelinating AEs reported. No significant differences in safety were noted between the adult and pediatric patients. ${ }^{34,35}$

Injection site and allergic reactions appear to be minimal. In parts 1 and 2 of the controlled study 31 patients reported having no injection-site reactions; 4 patients reported a mild reaction. In part 2 , a total of 13 of the 15 patients $(87 \%)$ receiving canakinumab and 15 of the 16 patients (94\%) receiving placebo reported no injection-site reactions. None were severe or led to discontinuation of treatment.

There are no data on safety in pregnancy in humans, although in extremely high doses given to marmoset monkeys and mice, delays in fetal skeletal development were found. No evidence of fetal malformations or embryonic toxicity was found in animal studies.

Several safety issues are in need of further investigation. Longer-term follow-up of greater numbers of patients is necessary. The lack of patients with classic NOMID is also noted. While in most cases of CAPS canakinumab is used as monotherapy, the safety of canakinumab combined with other immunosuppressant medications, used often for NOMID (at least prior to the use of anti-IL-1 therapies), may present additional safety hazards. The lack of head-to-head studies preclude direct comparison of different anti-IL-1 therapies but the safety profile of all appears similar, with perhaps more cases of vertigo and significantly fewer injection-site reactions with canakinumab.

\section{Patient satisfaction/acceptability}

In general patient satisfaction was remarkable with only 1 early withdrawal in the controlled study due to lack of efficacy. In the placebo withdrawal part of the controlled study four patients in the canakinumab group reported having severe symptoms associated with other disorders: 2 patients in 1 household had acute gastroenteritis, a third patient had painful fibromyalgia, and a fourth had migraine headaches. The effect on HRQoL was previously reported as well as the minimal injection-site reactions. Patients with MWS, especially those with hearing loss, need to be aware of the potential development of vertigo with treatment.

\section{Conclusions, place in therapy}

The efficacy of canakinumab in the treatment of CAPS provides proof of concept that IL- $1 \beta$ is an important factor in the pathogenesis of this disorder. Canakinumab given at $150 \mathrm{mg}$ per dose induced a complete response after the administration of a single dose in almost all the patients as well as inflammatory markers, and the effect appears to be sustained for up to at least 2 months between injections and continues to be effective with very little patient drop-out for longer periods. Some patients need higher doses (up to $300 \mathrm{mg}$ ) to induce a complete response. HRQoL and general mental and physical health and fatigue scores improved to the levels expected in the general population. The effect of canakinumab was similar in adults and children. To date, no deaths or life-threatening AEs have ocurred in CAPS patients treated with canakinumab and only mild AEs and symptoms were noted, none of which affected continuation of treatment. Still, there is a potentially higher risk for systemic infections and perhaps vertigo, especially in patients with MWS, which require special attention. The ability to administer canakinumab every 2 months with implications for compliance and minimal injection-site reactions is also attractive.

Other anti IL-1 medications (anakinra and rilonacept) also have demonstrated efficacy in CAPS..$^{18,19,28}$ However, the prolonged duration of action of canakinumab and low incidence of injection-site reactions may confer certain advantages for canakinumab, since both anakinra and rilonacept are frequently associated with injection-site reactions, and both require more frequent administration (daily for anakinra and weekly for rilonacept). ${ }^{18,19,28}$ However, 
unlike anakinra, there is still a relative lack of data on the effect of canakinumab in NOMID, especially on CNS manifestations and whether adjustments in doses and frequency are needed. A potential downside of canakinumab that has not been demonstrated in studies performed to date include the prolonged and profound neutralization of IL-1 and possible effects on infections or the response of the innate immune systems to infections. This issue necessitates further safety monitoring in long-term follow-up studies and post-marketing registries.

In conclusion, the dramatic role of canakinumab in the treatment of CAPS has been well demonstrated, particularly in patients with FCAS and MWS.

\section{Disclosures}

The authors declare no conflicts of interest.

\section{References}

1. Aksentijevich I, Putnam CD, Remmers EF, et al. The clinical continuum of cryopyrinopathies: novel CIAS1 mutations in North American patients and a new cryopyrin model. Arthritis Rheum. 2007;56:1273-1285.

2. Kile RL, Rusk HA. A case of cold urticaria with an unusual family history. JAMA. 1940;114:1067-1068.

3. Hoffman H, Mueller J, Brodie D, et al. Mutation of a new gene encoding a putative pyrin-like protein causes familial cold autoinflammatory syndrome and Muckle-Wells syndrome. Nat Genet. 2001;29:301-305.

4. Hoffman HM, Wanderer AA, Broide DH. Familial cold autoinflammatory syndrome: phenotype and genotype of an autosomal dominant periodic fever. J Allergy Clin Immunol. 2001;108:615-620.

5. Muckle TJ, Wells M. Urticaria, deafness and amyloidosis: a new heredo-familial syndrome. QJ Med. 1962;31:235-248.

6. Hawkins PN, Lachmann HJ, Aganna E, McDermott MF. Spectrum of clinical features in Muckle-Wells syndrome and response to anakinra. Arthritis Rheum. 2004;50:607-612.

7. Prieur AM, Griscelli G. Arthropathy with rash, chronic meningitis, eye lesions and mental retardation. J Pediatr. 1981;99:79-83.

8. Feldmann J, Prieur AM, Quartier P, et al. Chronic infantile neurological cutaneous and articular syndrome is caused by mutations in CIAS1, a gene highly expressed in polymorphonuclear cells and chondrocytes. Am J Hum Genet. 2002;71:198-203.

9. Aksentijevich I, Nowak M, Mallah M, et al. De novo CIAS1 mutations, cytokine activation, and evidence for genetic heterogeneity in patients with neonatal-onset multisystem inflammatory disease (NOMID): a new member of the expanding family of pyrin-associated autoinflammatory diseases. Arthritis Rheum. 2002;46:3340-3348.

10. Dinarello CA. Mutations in cryopyrin: bypassing roadblocks in the caspase-1 inflammasome for IL-1 $\beta$ secretion and disease activity. Arthritis Rheum. 2007;56:2817-2822.

11. Gattorno M, Tassi S, Carta S, et al. Pattern of IL- $1 \beta$ secretion on response to lipopolysaccharide and ATP before and after IL-1 blockade in patients with CIAS1 mutations. Arthritis Rheum. 2007;56:3138-3148.

12. Hoffman HM, Rosengren S, Boyle DL, et al. Prevention of coldassociated acute inflammation in familial cold autoinflammatory syndrome by interleukin-1 receptor antagonist. Lancet. 2004;364: 1779-1785.

13. Leslie KS, Lachmann HJ, Bruning E, et al. Phenotype, genotype, and sustained response to anakinra in 22 patients with autoinflammatory disease associated with CIAS-1/NALP3 mutations. Arch Dermatol. 2006;142:1591-1597.
14. Gerard S, le Goff B, Maugars Y, et al. Lasting remission of a Muckle-Wells syndrome with CIAS-1 mutation using half-dose anakinra. Joint Bone Spine. 2007;74:659.

15. Dalgic B, Egritas O, Sari S, Cuisset L. A variant Muckle-Wells syndrome with a novel mutation in CIAS1 gene responding to anakinra. Pediatr Nephrol. 2007;22:1391-1394.

16. Maksimovic L, Stimemann J, Caux F, et al. New CIAS1 mutation and anakinra efficacy in overlapping of Muckle-Wells and familial cold autoinflammatory syndromes. Rheumatology. 2008;47:309-310.

17. Kuemmerle-Deschner JB, Bialkowski A, Tzaribachev N, Kotter I. Anakinra in Muckle-Wells syndrome (MWS) - experience up to 29 months [abstract]. Clin Exp Rheumatol. 2008;26:204.

18. Goldbach-Mansky R, Shroff SD, Wilson M, et al. A pilot study to evaluate the safety and efficacy of the long-acting interleukin-1 inhibitor rilonacept (interleukin-1 Trap) in patients with familial cold autoinflammatory syndrome. Arthritis Rheum. 2008;58:2432-2442.

19. Hoffman HM, Throne ML, Amar NJ, et al. Efficacy and safety of rilonacept (interleukin-1 trap) in patients with cryopyrin-associated periodic syndromes. Arthritis Rheum. 2008;58:2443-2452.

20. Lachmann HJ, Kone-Paut I, Kuemmerle-Deschner JB, et al; for the Canakinumab in CAPS Study Group. Use of canakinumab in the cryopyrin-associated periodic syndrome. $N$ Engl J Med. 2009; 360:2416-2425.

21. Rynne M, Maclean C, Bybee A, et al. Hearing improvement in a patient with variant Muckle-Wells syndrome in response to interleukin 1 receptor antagonism. Ann Rheum Dis. 2006;65:533-534.

22. Mirault T, Launay D, Cuisset L, et al. Recovery from deafness in a patient with Muckle-Wells syndrome treated with anakinra. Arthritis Rheum. 2006;54:1697-1700.

23. Yamazaki T, Masumoto J, Agematsu K, et al. Anakinra improves sensory deafness in a Japanese patient with Muckle-Wells syndrome, possibly by inhibiting the cryopyrin inflammasome. Arthritis Rheum. 2008;58:864-868.

24. Federico G, Rigante D, Pugliese AL, et al. Etanercept induces improvement of arthropathy in chronic infantile neurological cutaneous articular (CINCA) syndrome. Scand J Rheumatol. 2003;32:312-314.

25. Lovell DJ, Bowyer SL, Solinger AM. Interleukin-1 blockade by anakinra improves clinical symptoms in patients with neonatal-onset multisystem inflammatory disease. Arthritis Rheum. 2005;52:1283-1286.

26. Rigante D, Ansuini V, Caldarelli M, et al. Hydrocephalus in CINCA syndrome treated with anakinra. Childs Nerv Syst. 2006;22:334-337.

27. Frenkel J, Wulffratt NM, Kuis W. Anakinra in mutation-negative NOMID/CINCA syndrome: comment on the articles by Hawkins et al. and Hoffman and Patel. Arthritis Rheum. 2004;50:3738-3739.

28. Goldbach-Mansky R, Dailey NJ, Canna SW, et al. Neonatal-onset multisystem inflammatory disease responsive to interleukin-1 beta inhibition. N Engl J Med. 2006;355:581-592.

29. Lachmann HJ, Lowe P, Daniela Felix S, et al. In vivo regulation of interleukin-1 beta in patients with cryopyrin-associated periodic syndromes. J Exp Med. 2009;206:1029-1036.

30. Church LD, McDermott MF. Canakinumab, a fully-human $m A b$ against IL-1 $\beta$ for the potential treatment of inflammatory disorders. Curr Opin Mol Ther. 2009;11:81-89.

31. Novartis: Ilaris prescribing information: http://www.pharma.us.novartis. com/product/pi/pdf/ilaris.pdf Accessed Feb 17, 2010.

32. Kuemmerle-Deschner JB, Tzaribachev N, Hansmann S, et al. Long-lasting response to ACZ885 (a new human IgG1 anti-IL-1 $\beta$ monoclonal antibody) in patients with Muckle-Wells Syndrome (MWS) [abstract]. Clin Exp Rheumatol. 2008;26:180.

33. Lachman H, Jung T, Felix S, et al. Treatment of cryopyrin associated periodic fever syndrome with a fully human anti-IL1beta monoclonal antibody (ACZ885): results from a subcutaneous administration study [abstract]. Clin Exp Rheumatol. 2008;26:181.

34. Kuemmerle-Deschner JB, Lachmann HJ, Hachulla E, et al. Efficacy and safety of canakinumab (Ilaris) in a large cohort of patients across different severity phenotypes of cryopyrin associated periodic syndrome (CAPS) [abstract]. Arthritis Rheum. 2009;60 Suppl 10:1235. 
35. Kuemmerle-Deschner JB, Cartwright R, Gattorno M, et al. Safety and efficacy of canakinumab (Ilaris) in children across different disease severity phenotypes of cryopyrin associated periodic syndrome (CAPS): interim results of an ongoing study [abstract]. Arthritis Rheum. 2009; 60 Suppl 10:2056.

36. Thornton BD, Hoffman HM, Bhat A, Don BR. Successful treatment of renal amyloidosis due to familial cold autoinflammatory syndrome using an interleukin 1 receptor antagonist. Am J Kidney Dis. 2007; 49:477-481.
37. Paul SM, Hildenbrand H, Jain MS, et al. Functional outcomes of one year of treatment of neonatal-onset multisystem inflammatory disease (NOMID) with Anakinra [abstract]. Arthritis Rheum. 2007; 56 Suppl:S291.

38. Lachmann HJ, Kone-Paut I, Kuemmerle-Deschner J, et al. Improved health-related quality of life in patients with cryopyrin-associated periodic fever syndrome (CAPS) after treatment with canakinumab (Ilaris), a fully human anti-IL-1 $\beta$ monoclonal antibody [abstract]. Arthritis Rheum. 2009;60 Suppl 10:1482.

\section{Publish your work in this journal}

Biologics: Targets \& Therapy is an international, peer-reviewed journal focusing on the patho-physiological rationale for and clinical application of Biologic agents in the management of autoimmune diseases, cancers or other pathologies where a molecular target can be identified. This journal is indexed on PubMed Central, CAS,

\section{Dovepress}

EMBase, Scopus and the Elsevier Bibliographic databases. The manuscript management system is completely online and includes a very quick and fair peer-review system, which is all easy to use. Visit http://www.dovepress.com/testimonials.php to read real quotes from published authors. 